\title{
Assessment of Neonatal Thermal Cares: Practices and Beliefs Among Rural Women in West Guji Zone, South Ethiopia: A Cross-Sectional Study
}

Wako Golicha Wako ( $\sim$ bwakot730@gmail.com )

Bule Hora University

Belda Negesa Beyene

Bule Hora University

\section{Research Article}

Keywords: Newborn, Thermal care, practice, beliefs, Ethiopia

Posted Date: December 3rd, 2020

DOl: https://doi.org/10.21203/rs.3.rs-114069/v1

License: (c) (i) This work is licensed under a Creative Commons Attribution 4.0 International License.

Read Full License 


\section{Abstract}

Introduction: A newborn has limited capacity to maintain temperature when exposed to cold environments. Therefore it is important to maintain newborn in optimal thermal environment. Neonatal hypothermia carries higher case fatality rate when concurrently occurs with other neonatal problems. Neonatal hypothermia is common neonatal problem.

Objectives: This study assessed neonatal thermal care practices and beliefs among rural women in west Guji Zone, south Ethiopia.

Methods and Materials: A community based cross sectional study combined with qualitative study was undertaken in rural areas of west Guji Zone. Randomly selected 388 rural mothers of infants less than 6 months old were participated in quantitative study. Three focus group discussions and three in-depth interviews were conducted among mothers of infants less than 6 months old; and grandmothers and traditional birth attendants respectively. Quantitative data was collected by using structured and pretested Afaan Oromo version questionnaire adapted from relevant literatures. Qualitative data was collected by in-depth interviews and focus group discussions. The qualitative data was cleaned, coded and analyzed by SPSS version 20 . Qualitative data was analyzed by thematic approach.

Results: Out of 423 women planned for the study, 388 women were successfully interviewed and included into the analysis. In general rural women believe in importance of newborn thermal protection. The findings show that approximately $75 \%$ and $85 \%$ of women dried and wrapped their newborns respectively after delivery. However in study area drying and wrapping newborn are usually done after first newborn bath. Just over $84 \%$ of women bathed their newborns in less than 6 hours of delivery and majority of them used warm water for the bathing. About $69.1 \%$ and $57.7 \%$ of women put head cover to their newborns immediately after birth and initiated breast feeding within one hour of delivery respectively. Skin to skin care of newborn is non-existent in study area and perceived as odd, frightening and potential dangerous practice.

Conclusion: Studied women practice some of recommended neonatal thermal care and believe in their importance in keeping newborn warm. However practice and believes about delayed first bath is against standard recommendation, whereas skin to skin care is non-existent and perceivably odd practice.

\section{Background}

A newborn has limited capacity to adapt to a range of environmental temperatures. A newborn has limited ability to dissipate heat effectively in warm environments. More critically, a newborn is unable to maintain temperature when exposed to cold environments. Therefore it is important to keep newborns in optimal thermal environment[1]. Neonatal hypothermia occurs when body temperature drops below lower limit of normal range of $36.5-37.5^{\circ} \mathrm{C}$. A newborn with temperature range of $36-36.4^{\circ} \mathrm{C}$ may be under cold stress; $32-35.9^{\circ} \mathrm{C}$ has moderate hypothermia and below $32^{\circ} \mathrm{C}$ is considered to be in severe hypothermia [2]. 
Neonatal hypothermia is a common problem of newborn and in some settings, as high as half of all newborns become hypothermic immediately after birth $[3,4]$. Mild to moderate hypothermia is nearly universal, with substantially higher risk in the cold season. However, incidence in the hot season is also high; thus, year-round thermal care promotion is required[5]. Neonatal hypothermia is one of major public health problems in Sub-Saharan Africa [6]. In Africa, prevalence of newborn hypothermia is high ranging from $44 \%$ to $85 \%[7]$. Though all newborns are at risk of losing heat after birth; preterm and/or small for gestational age are particularly vulnerable to hypothermia[7]. In Sub-Saharan Africa, $12.3 \%$ of newborns are born preterm and $25.5 \%$ of newborns are born small for gestational age (SGA[8].

Global case fatality rate of neonatal hypothermia ranges from 8.5 to $52 \%$; and the fatality rate is higher when hypothermia concurrently occurs with other neonatal problems[7]. Hypothermia rarely causes death, however it significantly contributes to neonatal mortality if concurrently occurred with severe infections, asphyxia and preterm birth[7]. It is associated with nearly fivefold increased risk of deaths within first 7 days of life[9]. With increasing importance of neonatal deaths and its sluggish reduction process, reduction in child mortality demands faster reduction neonatal death $[10,11]$. Thermal protection could be an important intervention to attain faster reduction of neonatal deaths. Three thermal care practices (delayed bathing, head covering and skin to skin care) could avert up to $20 \%$ of neonatal deaths caused by preterm birth complications and $10 \%$ of deaths in moderate to full term neonates[12]. In sub-Saharan Africa, importance of keeping newborns warm is well recognized[13]. However neonatal thermal care practice is suboptimal in the region[13]. Harmful cultural norms and traditional practices that affect neonatal thermal care practices at home exist in different contexts of Africa[14]. The lack of thermal protection is still an unrecognized major challenge for newborn survival in developing countries[7]. Few studies have assessed neonatal thermal care practices in rural Ethiopia. Thus this study assessed practices and beliefs of rural women about neonatal thermal cares (early drying and wrapping, head covering, skin to skin care, newborn bath time and early breast feeding initiation) in West Guji Zone, South Ethiopia.

\section{Methods And Materials}

\section{Study Setting and Period}

The study was conducted in West Guji zone, South Ethiopia from October 2018 to April 2019. West Guji zone's administrative town, Bule Hora is located $475 \mathrm{~km}$ south to Ethiopia's capital, Addis Ababa; at latitude and longitude of $5^{0} 35^{\prime} \mathrm{N} 38^{0} 15^{\prime} \mathrm{E} / 5.583^{\circ} \mathrm{N} 38.250^{\circ} \mathrm{E}$ respectively. The town is location at an elevation of 1716 meters above sea level and has average annual rainfall of $648 \mathrm{~mm}$. According to 2007 Ethiopian census, the town has a total population of 27,820 .

\section{Study Design}

A community based cross sectional study combined with qualitative study was done. We conducted quantitative cross-sectional study to assess neonatal thermal care practices. Focus groups discussions 
and in-depth interviews were conducted to supplement quantitative assessment and; to assess rural women's beliefs about neonatal thermal care practices.

\section{Study population}

Participants of quantitative study were rural women who has infant of six months old or younger infants. Focus group discussions were conducted among rural women who has infant of six months old or younger. In-depth interviews were conducted among grandmothers and traditional birth attendants.

\section{Exclusion criteria}

We excluded women who gave birth to their last infant by cesarean section and/or underwent major surgical interventions immediately after delivery from quantitative study. We excluded them, because such surgical interventions may compromise women's ability to recall thermal cares provided to their newborns immediately after delivery.

\section{Study variables}

We assessed neonatal thermal cares focusing on practices and beliefs of rural women as related to immediately drying and wrapping, head covering, skin to skin care, time of newborn baths, time of breast feeding initiation after delivery.

\section{Sample size calculation}

Sample sizes for the first six objectives were calculated by single proportion sample size calculation formula with the following common assumptions:

- An anticipated sample proportion ( $\mathrm{p}$ ) of $50 \%$. As there is no previous similar study done in rural Ethiopia. We assumed prevalence of early wrapping and drying; delayed newborn baths at least by 6 hours; head covering during neonatal period, skin to skin care, breastfeeding initiation within 1 hour of delivery to be $50 \%$;

- Level of confidence of $95 \%$ and

- Margin of error (w) of $5 \%$.

Then the sample size was calculated using the formula: $n=Z^{2} p(1-p) / w^{2}$;

Where $\mathrm{n}$ - is minimum required sample size; $\mathrm{Z}$ - is z-score corresponding to two sided $95 \%$ confidence level; $\mathrm{P}$ - is anticipated sample proportion and $\mathrm{w}$ - is margin of error. After imputing the numbers into the formula the minimum required sample sizes was 384 women. Then adding $5 \%$ non-response rate, the final require sample size was $384+384(0.05)=423$ women. This sample size was planned for quantitative study.

Total of 3 focus group discussions (FGDs) were planned to be conducted; one focus group discussion from each of three climatic areas of the zone (i.e Kola, Woindega and Dega climate areas). All FGDs had 
8-10 participants. Similarly total of 3 in-depth interviews (IDIs) were planned to be conducted; one IDI from each of three climatic areas of the zone.

\section{Sampling procedures}

Sample size of quantitative study was distributed to three climatic zones within the study area proportional to the approximate population of each climatic zones. From each climatic area, one kebele (i.e the smallest administrative unit) was randomly selected. List of all eligible women within selected kebeles were collected from health posts within the kebeles. Then required sample sizes from each kebele were randomly selected from list of eligible women within the kebele.

Participants of FGDs were purposively selected to ensure variation in age, socio-economic status and level of education. Key informants of IDIs were selected purposively based on judged depth of information that can be obtained.

\section{Data collection instruments and procedures}

Quantitative data was collected by using structured and pretested Afaan Oromo (i.e local language of the study area) questionnaire (file1) adapted from relevant literatures. Data collectors were high school graduate local women who fluently speak Afaan Oromo. Quantitative data was collected at home of mothers. Focus group discussions and in-depth interviews were conducted by investigators. Focus group and in-depth interview guides (file2 and file3) prepared in local language (i.e Afaan Oromo) were used to facilitate focus group discussions and in-depth interviews. Focus group discussions and in-depth interviews were tape recorded.

\section{Quality assurance}

Training was provided for data collectors and supervisors. Investigators supervised all quantitative data collection process. Questionnaire was pretested on population with comparable socio-demographic characteristics with the study population outside the sites where the current study was conducted. Adapted English questionnaire was converted into Afaan Oromo language and reconverted back into English by two independent language experts who were judged to be fluent in both languages. All necessary inputs from pretest were incorporated into final questionnaire.

\section{Data processing and analysis}

Quantitative data was entered into Epi-info 7 and transferred to Statistical Package for Social Sciences (SPSS) version 20 for further processing. The data was be cleaned, coded and analyzed by SPSS version 20. Frequency of different thermal care practices was generated. Qualitative data was transcribed, translated and entered into open code software. The qualitative data was coded by themes and analyzed by thematic approach.

\section{Results}




\section{Socio-demographic characteristics of respondents}

Out of the total 423 reproductive age women planned for the study, 388 women were successfully interviewed with the response rate of $91.7 \%$. All of the 388 interviewed women were included in the final analysis. The mean $( \pm S D)$ age of the respondents was $25.4( \pm 5)$ years. At the time of data collection, the mean $( \pm S D)$ age of the infants about which thermal care was assessed is $3.8( \pm 1.6)$ months. Majority of women were literate (i.e. can read and write), which accounted for $283(72.9 \%)$ and the rest $105(27.1 \%)$ were illiterate (i.e. cannot read and write). Protestant Christian was the commonest religion; followed by Islam, Orthodox Christian and Waqefata (i.e traditional belief); each accounted 251(64.7\%), 40(10.3\%), $36(9.3 \%)$ and $36(9.3 \%)$ respectively. Regarding the marital status of women, at the time of interview; 325(83.8\%) were married, 25(6.4\%) were widowed, 17(4.4\%) were separated, and 16(4.1\%) were divorced. Table 1 presents socio-demographic characteristics of the respondents.

Table 1. Socio-demographic characteristics of the respondents, West Guji Zone, Oromia; Southern Ethiopia; June 2020 


\begin{tabular}{|c|c|c|}
\hline Characteristics of respondents & Frequency & Percentage (\%) \\
\hline \multicolumn{3}{|l|}{ Age of women in completed years $(n=388)$} \\
\hline$<18$ & 9 & 2.3 \\
\hline $18-34$ & 345 & 88.9 \\
\hline $35-49$ & 34 & 8.8 \\
\hline \multicolumn{3}{|l|}{ Women's educational level $(n=388)$} \\
\hline No education(grade 0 ) & 126 & 32.5 \\
\hline Primary education(grade 1-8) & 221 & 56.9 \\
\hline Secondary education and above (grade 9 and above ) & 41 & 10.6 \\
\hline \multicolumn{3}{|l|}{ Women's occupation(n=388) } \\
\hline Farmer & 165 & 42.5 \\
\hline House wife & 75 & 19.4 \\
\hline Merchant & 105 & 27.1 \\
\hline Daily laborer & 23 & 5.9 \\
\hline Employed & 16 & 4.1 \\
\hline others & 4 & 1.0 \\
\hline \multicolumn{3}{|l|}{ Women's Religion $(n=388)$} \\
\hline Waqefata & 36 & 9.3 \\
\hline Protestant Christian & 251 & 64.7 \\
\hline Orthodox Christian & 36 & 9.3 \\
\hline Islam & 40 & 10.3 \\
\hline Catholic Christian & 23 & 5.9 \\
\hline Others & 2 & 0.5 \\
\hline \multicolumn{3}{|l|}{ Sex of the youngest infant } \\
\hline Male & 213 & 54.9 \\
\hline Female & 175 & 45.1 \\
\hline
\end{tabular}

\section{Past Obstetrics History of Studied Women}


At the time of survey, majority of the respondents, 311(80.1\%) were women who gave birth more than once. Significant proportion of women, 250(64.4\%) delivered the youngest infant at home and the remaining $138(35.6 \%)$ of women delivered in health facilities. Among these deliveries, only $120(30.9 \%)$ of them were attended by health workers of variable level of qualification. However, majority of these women, 298(76.8\%) attended antenatal care during pregnancy of the last infant. Table 2 presents past obstetric history of study participants.

Table 2. Past obstetrics history of rural women, West Guji Zone, Oromia; southern Ethiopia, June 2020 


\begin{tabular}{|c|c|c|}
\hline Past obstetrics history & Frequency & Percentage (\%) \\
\hline \multicolumn{3}{|c|}{ Number of all previous births $(n=388)$} \\
\hline 1 & 77 & 19.8 \\
\hline $2-4$ & 187 & 48.2 \\
\hline$>=5$ & 124 & 31.9 \\
\hline \multicolumn{3}{|c|}{ Did you seek ANC during pregnancy of the most recent birth $(n=388)$} \\
\hline No & 90 & 23.2 \\
\hline Yes & 298 & 76.8 \\
\hline \multicolumn{3}{|c|}{ Place of the birth of the last infant $(n=388)$} \\
\hline Home & 250 & 64.4 \\
\hline Health post & 22 & 5.7 \\
\hline Health center & 28 & 7.2 \\
\hline Hospital & 84 & 21.6 \\
\hline Others & 4 & 1.0 \\
\hline \multicolumn{3}{|c|}{ Attendants of the last birth $(n=388)$} \\
\hline Traditional birth attendant & 38 & 9.8 \\
\hline Relative & 162 & 41.7 \\
\hline HEW* & 40 & 10.3 \\
\hline Health professional & 80 & 20.6 \\
\hline Nobody & 65 & 16.7 \\
\hline Others & 3 & 0.8 \\
\hline \multicolumn{3}{|c|}{ Gestational age of the last infant $(n=388)$} \\
\hline$<9$ months & 6 & 1.5 \\
\hline >=9 months & 382 & 98.5 \\
\hline
\end{tabular}

*: HEW- Health Extension Worker.

\section{Women's Practice of Neonatal Thermal Care}

Majority, 350(90.2\%) of women had prepared clothes for immediate drying of babies before delivery. However, only $32(8.2 \%)$ of women dried-off their babies of birth fluids before delivery of placenta. Significant proportion of women, $131(33.8 \%)$ and 135(35.3\%) performed drying of their babies 
immediately and long time after placenta delivery respectively. A qualitative finding shows that drying is usually done after first bath. Focus group discussants said like this "immediately after delivery before drying, newborns are bathed and then wrapped with clean clothes. But if a delivery is at a health facility, newborns are not bathed. They are wrapped with clothes and no bath is done until mothers return their home from the health facility"- 18 years old and mother of 5 months old infant... "Some women do drying and wrapping. But this practice is at its early state of expansion among women who have exposure to health facilities. The common practice is to bath the baby immediately after delivery with warm water and, then wrap with warm clothes" -18 years old and mother of 6 months old infant.

Majority, 336(86.6\%) of women have prepared clothes for immediate wrapping of their baby before delivery. However, only $113(8.2 \%)$ of women wrapped their babies of within 5 minutes of delivery. Table 3 presents women's practice of immediate drying and wrapping of newborns.

\section{Table 3. Newborn immediate drying and wrapping practices among rural women in West Guji Zone, Oromia; southern Ethiopia, June 2020}




\begin{tabular}{|c|c|c|}
\hline Newborn drying and wrapping practices & Frequency & Percentage (\%) \\
\hline \multicolumn{3}{|l|}{ First drying time $(n=388)$} \\
\hline Immediately before placental delivery & 32 & 8.2 \\
\hline Immediately after placental delivery & 131 & 33.7 \\
\hline Long time after placental delivery & 137 & 35.3 \\
\hline Cannot remember & 88 & 22.7 \\
\hline \multicolumn{3}{|l|}{ First drying time in minute $(n=388)$} \\
\hline In less than 5 minutes & 92 & 23.7 \\
\hline Between 5-15 minutes & 125 & 32.2 \\
\hline Between 16-30 minutes & 51 & 13.1 \\
\hline After 30 minutes & 57 & 14.7 \\
\hline Could not remember & 63 & 16.2 \\
\hline \multicolumn{3}{|l|}{ Reason for delayed drying $(n=233)$} \\
\hline attendant was focusing on mothers until placenta delivery & 80 & 34.3 \\
\hline Waited until umbilical cord is cut & 17 & 7.3 \\
\hline Waited until placental delivery & 74 & 31.8 \\
\hline Drying baby is taboo until placental burial & 27 & 11.6 \\
\hline Nobody was available & 21 & 9.0 \\
\hline Waited until baby get bath & 14 & 6.00 \\
\hline \multicolumn{3}{|l|}{ First wrapping time in minute $(\mathrm{n}=388)$} \\
\hline In less than 5 minutes & 113 & 29.1 \\
\hline Between 5-15 minutes & 68 & 17.5 \\
\hline Between 16-30 minutes & 38 & 9.8 \\
\hline After 30 minutes & 117 & 30.1 \\
\hline Could not remember & 52 & 13.4 \\
\hline \multicolumn{3}{|l|}{ Reason for delayed wrapping( $n=223)$} \\
\hline attendant was focusing on mothers until placenta delivery & 45 & 20.2 \\
\hline Waited until umbilical cord is cut & 28 & 12.5 \\
\hline Waited until placental delivery & 43 & 19.3 \\
\hline
\end{tabular}




\begin{tabular}{|lll|}
\hline Wrapping baby is taboo until placental burial & 6 & 2.7 \\
\hline Nobody was available & 15 & 6.7 \\
\hline Waited until baby get bath & 86 & 38.6 \\
\hline
\end{tabular}

Great majority, 328(84.5\%) of women have bathed their baby in less than 6 hours of delivery and $306(78.9 \%)$ women used warm water for the first bath. Forty two $(70.0 \%)$ of women who delayed first newborn bath at least by 6 hours after delivery did so because they were advised by health workers advised them to do so, whereas only $8(11.7 \%$ of delayed bath) women delayed the bath because it keeps the newborns warm. Table 4 presents newborn first bath time among rural women in west Guji Zone.

Table 4. Rural women's practice of first newborn bath in west Guji Zone, Oromia; southern Ethiopia, June 2020

\begin{tabular}{|lll|}
\hline First newborn bath practices & Frequency & Percentage (\%) \\
\hline First bath time after delivery ( $\mathbf{n = 3 8 8 )}$ & 328 & 84.5 \\
\hline In less than 6 hours & 60 & 15.5 \\
\hline after 6 hours & & \\
\hline Type of water used for first bath(=388) & 306 & 78.9 \\
\hline Warm water & 61 & 15.7 \\
\hline Cold water & 21 & 5.4 \\
\hline others & & \\
\hline Reason for delayed newborn bath(n=60) & 8 & 13.3 \\
\hline To keep baby warm & 42 & 70.0 \\
\hline Health professional advised me to do so & 10 & 16.7 \\
\hline Others & & 1.2 \\
\hline Reason for Early bathing (n=328)* & 270 & 82.3 \\
\hline To remove different secretions or blood or dirty from body & 27 & 18.9 \\
\hline To remove visible vernix & 62 & 5.5 \\
\hline To improve health and strength of newborn & 18 & 7.9 \\
\hline To make baby refreshed and comfortable & 26 & 4.6 \\
\hline Encouraging sleep and improving health & 15 & 10.1 \\
\hline To prevent different smells & 33 & 1.2 \\
\hline Others & & \\
\hline
\end{tabular}


*- the percentage may not add up to 100 as responses are mutual exclusive

Two hundred sixty eight(69.1\%) women put head covering to their baby immediately after delivery and almost all of them, $267(68.8 \%)$ maintained the practice during neonatal period. Two hundred sixty six $(68.6 \%)$ women have prepared head covering when nearing term.

Three hundred sixty four (93.8\%) women breastfed their baby and $210(57.7 \%)$ of them initiated breast feeding within one hour of delivery. Exclusive breast feeding during neonatal period was practiced by $227(62.4 \%)$ women. Table 5 presents rural women's breast feeding practice after delivery in west Guji.

Table 5. Rural women's practice of breast feeding during neonatal period, West Guji Zone, Oromia; southern Ethiopia, June 2020 
Time to initiate breast feeding after delivery $(n=364)$

Within one hour

after one hour

What did you do with colostrum?(=364)

Provided to baby

Discarded

others

Provided extra foods/fluids during neonatal period $(n=388)$

Yes

137

227

24

Could not remember

\section{Reasons for delayed initiation of breast feeding within 1 hour of delivery $(n=154)$}

Colostrum is dirty/not good for baby

Lack of sufficient breast milk

Baby need sleep/rest after delivery

Baby did not show sign of hunger

Baby needed to be bathed

Mother needed rest

Mother needed bathing

Others
210

154

136

226

2
0.6

62.1

0.6


months old infant...."Early bathing and then wrapping with clean clothes prevent cold"-18 years old and mother of 6 months old infant. Regarding immediate drying of newborn which is usually done after first bath, women believes that drying with dry clothes damages fragile skin of newborns. A focus group discussant quoted "because newborn's skin is very fragile, it should not touched by clothes for drying. That is why bath is done to remove body secretions"-36 years old and mother of 6 months old infant.

Early bathing of newborns as soon as possible after delivery of placental is deep rooted practice. Women strongly believe that newborns should be bathed as soon as possible after delivery provided that placental delivery is not delayed. Other recommended newborn thermal cares like immediate drying and wrapping are usually done after the first newborn's bath opposite to the standard recommendation. However newborn's bath is rarely done before delivery of placenta. Focus discussants quoted "if placenta is safely delivered, the newborns should be bathed immediately and kept away from cold'-25 years old and mother of 5 months old infant... "If placenta is immediately delivered, then the baby will be bathed immediately. Then there will be no problem of cold'- 22 years old and mother of 6 months old infant.

All women believe in importance of head covering to prevent cold from newborns. Also immediate covering of head of newborns as soon as possible is believed to be important practice. Focus group discussants quoted "Yes it is helpful. It prevents cold from injuring baby"-33 years old and mother of 5 months old infant... "Head covering should be done immediately after delivery"- 28 years old and mother of 6 months old infant.

Initiation of breastfeeding is almost universal and women claim that they initiate breast feeding immediately after placental delivery and the first bath. However they did not mention or believed in thermal care importance of either early or frequent breast feeding. Focus group discussants quoted "I do not think that early initiation of breast feeding benefits in keeping baby warm except that delayed initiation of breastfeeding results in baby's failure to familiarize with breastfeeding"-20 years old and mother of 6 months old infant... "Early initiation of breastfeeding does not prevent cold from newborns. Delaying up to 2to 3 hours after delivery without initiating breastfeeding does not have any problem. Just we learned early initiation of breastfeeding from our mothers"-25 years old and mother of 5 months old infant.

Skin to skin care of newborn is non-existent and strange thermal care practice in study area. Women perceive skin to skin care of newborn as an odd, frightening and potential dangerous ways of handling newborns. Focus group discussants quoted "such practice (i.e skin to skin care) is not done in this area. Holding baby in such way is frightening. This practice is new to us. We hear/see it today"-20 years old and mother of 6 months old infant... "If skin to skin care is done, babies may get injured especially by inexperienced young mothers"- 30 years old and mother of 4 months old infant... "Because we are strange to it (i.e skin to skin care), it looks somewhat odd to us"- 20 years old and mother of 6 months old infant. Elder women would not advise younger women to do skin to skin care. Furthermore, women do not believe that skin to skin care helps in keeping newborn warm. However they claimed that they can do skin to skin care if taught to do so. 


\section{Discussion}

Studied women collectively agree on importance of thermal protection of newborns. Drying, wrapping, head covering and early initiation of breastfeeding are common neonatal thermal care practices, whereas delayed bathing and skin to skin care are infrequent thermal care practices in study area. Local women believe that wrapping, head covering and early bath keep newborn warm. They do not believe early initiation of breastfeeding and skin to skin care help in keeping newborns warm.

In this study over $75 \%$ and $85 \%$ of women reported drying and wrapping newborns after delivery respectively. Approximately $23 \%$ and $29 \%$ newborns were dried and wrapped respectively within 5 minutes of delivery. This finding is somehow similar to a study conducted in Tanzania where more than $75 \%$ of newborns were dried and wrapped after delivery[15]. In Tanzania, $42 \%$ and $27 \%$ of newborns were dried and wrapped within five minutes of delivery respectively[15]. In south Asia, a study reported better drying and wrapping practice, where $81 \%$ of newborns in India; $66 \%$ of newborns in Bangladesh and $83 \%$ of newborns in Nepal receive immediate drying and wrapping[16]. In current study area, commonly drying and wrapping are done after newborn bath except in the circumstance of health facility delivery, where limited access to water and/or health workers' advice might have hindered early newborn bath. Though women accept general importance of wrapping in preventing cold, they believe better thermal protection would be achieved if drying and wrapping are preceded by bath.

Head covering reduces heat loss through large surface area of newborn's head[2]. Lack of head covering is significantly associated with neonatal hypothermia [17]. In current study, $69.1 \%$ of women covered their newborns' head immediately after delivery. Almost all of them (68.8\%) maintained the practice neonatal period and they believe that head covering is an important means of protecting newborns from cold.

Delayed initiation of breastfeeding is one of risk factors for neonatal hypothermia [18, 19]. More than half of women $(57.7 \%)$ initiated breast feeding within one hour of delivery and $37.4 \%$ of women fed colostrum to their baby. Similar findings were reported from a systematic review conducted in sub-Saharan Africa (Ethiopia included)[13]. In Tanzania, only 18\% of women initiated breastfeeding within one hour of delivery [15]. Studied women do not believe that early initiation of breastfeeding does help in keeping newborns warm. They simply initiated breastfeeding early because that was what they have learnt from their grandmothers/elder women.

Early bathing contributes to significant health loss and increases risk of hypothermia $[18,20]$. Delaying newborn bath by at least 6 hours helps in keeping newborns warm and consequently reduces risks of morbidity and mortality associated with neonatal hypothermia[7]. In this study, only $15.5 \%$ of women delayed first newborn bath by at least 6 hours. Previous study also reported that $70 \%$ newborns in Ethiopia were bathed within 6 hours of delivery[13]. Study conducted in Tanzania reported smaller figure where $59 \%$ of newborns were bathed within 6 hours of delivery[15]. In Zambia women participated on qualitative study stated that newborn are usually bathed immediately after delivery [21]. Similar to previous studies from Sub-Saharan Africa $[13,21]$, main reasons for early bathing are to remove different secretions/blood/dirty from newborn's body, to avoid bad smell and remove visible vernix. Studied 
women do not believe that delaying newborn bath helps in keeping newborn warm; instead they believe early bath helps in keeping newborn warm. This might be due fact that nearly $80 \%$ of women used warm water for bathing newborns.

Skin to skin care is non-existent, odd and strange practice in study area. Studies conducted in Sub Saharan Africa reported similar findings [13, 21]. In Zambia skin to skin care is not practiced[22]. Studies found, in Tanzania skin to skin care is new to local women and rarely practiced[21] [15]. In Ghana $10 \%$ of newborn received skin to skin care[23]. However higher prevalence of skin to skin care was reported from south Asian countries. In Bangladesh and Nepal about $25 \%$ and $38 \%$ of women reported practicing skin to skin care of newborns respectively [16]. Studied women do not believe that skin to skin care is helpful to keep newborn warm. Moreover they also perceive the practice as potential dangerous to newborn especially for younger mothers who do not have experience of handling delicate body of newborns. Current believe and perception surrounding skin to skin care might hinder future implementation of the practice in the area. Shamba et al also reported that possible danger to newborns delicate bone perceived by women as a main barrier to skin to skin care in Tanzania [21].

This study assessed neonatal thermal care practices of women who gave birth within 6 months preceding the survey date. Women were inquired to remember and report actual practices they did to provide thermal protection to their newborns within 6 months preceding the survey. Therefore recall bias might hinder accurate reporting of actual thermal care surrounding immediate postpartum period. This limitation should be taken into account when interpreting this report.

\section{Conclusion}

Studied women practice some of recommended neonatal thermal care and believe in their importance in keeping newborn warm. However practice and believes about delayed first bath is against standard recommendation, whereas skin to skin care is non-existent and perceivably odd practice. Immediate drying and wrapping are done by significant number of women, however the timing is not correct as they are done mostly after first newborn bath. Further study should be undertaken to acquire in-depth understanding of motives for early newborn bath.

\section{Abbreviations}

FGD: Focused Group Discussion

IDI: In-Depth Interview

IUGR: Intra Uterine Growth Restriction

MDG: Millennium Development Goal

SGA: Small for Gestational Age

Page $17 / 20$ 
TBA: Traditional Birth Attendant

WHO: World Health Organization

\section{Declarations}

\section{Ethics approval and consent to participate}

Ethical clearance was obtained from institutional review board of Bule Hora University. All methods were performed in accordance with a proposal approved by Bule Hora University. Informed written consent was obtained from all adult subjects. Informed verbal consent was obtained from parents or legal guardians for subjects less than 18 years old. Confidentiality of the information was secured by omitting personal modifiers on the questionnaire.

\section{Consent for publication}

Not applicable

\section{Availability of data and materials}

Full data set and other materials pertaining to this study can be obtained from corresponding author on reasonable request.

\section{Competing interest}

The authors declare that they have no competing interests

\section{Funding}

This research was financed by Bule Hora University. The funder did not have any role in design of the study, data collection, analysis, interpretation, writing manuscript and decision to publish

\section{Authors' contribution}

WG conceived the project, developed proposal, collected data, did data analysis and interpretation and wrote the manuscript. BN developed proposal and collected data. Both authors read and approved the final manuscript.

\section{Acknowledgements}

First of all, we thank Almighty God for giving us strength, patience and endurance during this research undertaking. Next, our thank goes to Bule Hora University for providing financial assistance to complete this research, without which this research could not have been realized. We also acknowledge health officials from district health offices within West Guji Zone and health workers, especially health extension workers who cooperate and provide assistance during field works. Their contributions were very 
immense. We are also grateful to all data collectors for their commitments. Lastly, but not the least we thank rural women who have participated in this study for their priceless time.

\section{Authors' Information}

Wako Golicha (MPH): is Assistant Professor at Bule Hora University College of Health and Medical Sciences, Ethiopia.

Belda Negesa (BSC): is Assistant Lecturer at Bule Hora University College of Health and Medical Sciences, Ethiopia.

\section{References}

1. Steven G. Gabbe, Jennifer R. Niebyl, and J.L. Simpson., Obstetrics: Normal and Problem Preganacies. 5th ed. 2007, Philadelphia, USA: Churchill Livingstone Elsevier;.

2. World Health Organization(WHO), Thermal Control of the Newborn:a Practical Guide. Hypothermia in Infants. 1994, World Health Organization: Geneva, Switzerland. p. 3-5.

3. F. Zayeri, A., et al., Incidence and Risk Factors of Neonatal Hypothermia at Referral Hopitals in Tehran, Islamic Republic of Iran. Eastern Mediterranean Health Journal, 2007. 13: p. 1308-18.

4. Luke C. Mullany, Joanne Katz, and Subarna K. Khatry et al, Incidence and Seasonality of Hypothermia Among Newborns in Southern Nepal. Arch Pediatr Adolesc Med, 2010. 164(1): p. 71-7.

5. Luke C. Mullany and Etal, Neonatal hypothermia and associated risk factors among newborns of southern Nepal. BMC Medicine, 2010. 8: p. 43.

6. R Onalo, Neonatal hypothermia in sub-Saharan Africa: A review. Nigerian Journal of Clinical Practice, 2013. 16(2).

7. Karsten Lunze, et al., The global burden of neonatal hypothermia: systematic review of a major challenge for newborn survival. BMC Medicine, 2013. 11: p. 24.

8. Anne CC Lee, et al., National and regional estimates of term and preterm babies born small for gestational age in 138 low-income and middle-income countries in 2010. Lancet, 2013. 1: p. e26-36.

9. Morten Sodemann, et al., Hypothermia of newborns is associated with excess mortality in the first 2 months of life in Guinea-Bissau, West Africa. Tropical Medicine and International Health, 2008. 13: p. 980-6

10. Jennifer Bryce, Cesar G Victora, and Robert E Black, The unfinished agenda in child survival. Lancet, 2013. 382: p. 1049-59.

11. Group., W.B., Disease Control Priorities. 2016, Washington DC: World Bank.

12. Zulfi qar A Bhutta, et al., Can available interventions end preventable deaths in mothers, newborn babies, and stillbirths, and at what cost? . Lancet, 2014. 384: p. 347-70.

13. Margaret Bee, A.S., Zelee Hill. , Neonatal care practices in sub-Saharan Africa: a systematic review of quantitative and qualitative data. Journal of Health, Population and Nutrition, 2018. 37: p. 9. 
14. Wendy S Coalter and Sarah L Patterson, Sociocultural factors affecting uptake of home-based neonatal thermal care practices in Africa: A qualitative review. Journal of Child Health Care, 2017. 21(2): p. 132-41.

15. Suzanne P. and Etal, A Large Cross-Sectional Community-Based Study of Newborn Care Practices in Southern Tanzania. PLoS One, 2010. 5(12): p. e15593.

16. Mallick, L., J. Yourkavitch, and C. Allen, Trends, determinants, and newborn mortality related to thermal care and umbilical cord care practices in South Asia. BMC Pediatr, 2019. 19(1): p. 248.

17. Alebachew Bayih, W., et al., Neonatal hypothermia and associated factors within six hours of delivery in eastern part of Ethiopia: a cross-sectional study. BMC Pediatr, 2019. 19(1): p. 252.

18. Tasew, H., et al., Determinants of hypothermia on neonates admitted to the intensive care unit of public hospitals of Central Zone, Tigray, Ethiopia 2017: unmatched case-control study. BMC Res Notes, 2018. 11(1): p. 576.

19. Demissie, B.W., et al., Neonatal hypothermia and associated factors among neonates admitted to neonatal intensive care unit of public hospitals in Addis Ababa, Ethiopia. BMC Pediatr, 2018. 18(1): p. 263.

20. Ukke, G.G. and K. Diriba, Prevalence and factors associated with neonatal hypothermia on admission to neonatal intensive care units in Southwest Ethiopia - A cross-sectional study. PLoS One, 2019. 14(6): p. e0218020.

21. Shamba, D., et al., Thermal care for newborn babies in rural southern Tanzania: a mixed-method study of barriers, facilitators and potential for behaviour change. BMC Pregnancy Childbirth, 2014. 14: p. 267.

22. Karsten Lunze and etal, Prevention and Management of Neonatal Hypothermia in Rural Zambia. PLoS ONE, 2014. 9(4): p. e92006.

23. Zelee Hill and et al, Keeping newborns warm: beliefs, practices and potential for behaviour change in rural Ghana. Tropical Medicine and International Health, 2010. 15(10): p. 1118-24.

\section{Supplementary Files}

This is a list of supplementary files associated with this preprint. Click to download.

- File1.docx

- File1.docx

- File2.docx

- File2.docx

- File3.docx

- File3.docx 